\title{
LA DIFERENCIAL NO ES UN INCREMENTO INFINITESIMAL. EVOLUCIÓN DEL CONCEPTO DE DIFERENCIAL Y SU CLARIFICACIÓN EN LA ENSEÑANZA DE LA FÍSICA
}

\author{
MARTÍNEZ TORREGROSA, J. ${ }^{1}$, LÓPEZ-GAY, R. ${ }^{1}$, GRAS MARTÍ, A. ${ }^{2}$ \\ y TORREGROSA GIRONÉS, G. ${ }^{3}$ \\ ${ }^{1}$ Departamento de Didáctica General y Didácticas Específicas \\ ${ }^{2}$ Departamento de Física Aplicada \\ ${ }^{3}$ Departamento de Análisis Matemático y Matemática Aplicada. Universidad de Alicante. \\ joaquin.martinez@ua.es, rlucio@clientes.unicaja.es, agm@ua.es
}

\begin{abstract}
Resumen. El uso de la diferencial en la enseñanza de la física en el último año de bachillerato y cursos universitarios es tan frecuente como poco comprendido. Para identificar el origen de esa situación y diseñar propuestas para superarla, se ha realizado un estudio histórico y epistemológico destinado a clarificar el significado y el papel que juega la diferencial de la física. Como resultado, se describen las aportaciones e insuficiencias de dos concepciones históricas (Leibniz y Cauchy), y se presenta con detalle una propuesta alternativa basada en la concepción del matemático francés Fréchet, formulada a principios del siglo xx. Como conclusión de este estudio, se enumeran un conjunto de indicadores de lo que sería una adecuada comprensión del concepto de diferencial en las clases de física.

Palabras clave. Diferencial, cálculo, física, matemáticas, enseñanza.
\end{abstract}

Summary. Despite its frequent use, there is little understanding of the concept of differential among upper high school and undergraduate students of Physics. As a first step to identify the origin of this situation and to design proposals to revert it, we have done a historic and epistemologic study aimed at clarifying the role and the meaning of the differential in Physics. We describe the contributions of Leibniz and Cauchy, and stress their shortcomings, which are overcome by the alternative definition proposed by the French mathematician Fréchet, dating from early XX century. As a result of this study, we conclude by outlining a set of indicators of what would be a proper understanding of the concept of differential in Physics education.

Keywords. Differential, calculus, physics, mathematics, education.

\section{INTRODUCCIÓN Y PLANTEAMIENTO DEL PROBLEMA}

El cálculo diferencial se empieza a utilizar en los textos y clases de física en el último año de bachillerato, y en el nivel universitario está presente en el desarrollo de la práctica totalidad de los tópicos de física. Esto es reflejo, sin duda, de la necesidad del cálculo para estudiar situaciones físicas mínimamente complejas, más cercanas a la realidad que las tratadas en los cursos elementales: la historia de la ciencia muestra cómo la invención del cálculo diferencial supuso un salto cualitativo en el tipo y complejidad de problemas que pudieron abordarse desde entonces (Aleksandrov et al., 1956; Edwards, 1937; Kline, 1972).

En contraste con esta importancia, las conclusiones de distintos trabajos, realizados generalmente en el ámbito de la enseñanza de las matemáticas, han puesto de 
manifiesto la existencia de serias deficiencias entre estudiantes, e incluso entre profesores, en relación con la comprensión de las ideas fundamentales del cálculo (Azcárate, 1990; Breitenberger, 1992; Ferrini-Mundy y Gaudard, 1992; Ferrini-Mundy y Geuther, 1991, Orton, 1983a, 1983b) y, más concretamente, en relación con el concepto de diferencial (Alibert et al., 1987; Artigue y Viennot, 1987). Por nuestra parte, hemos comprobado el bajo porcentaje de estudiantes de física de COU y primeros cursos de carreras científico-técnicas que usan el cálculo diferencial en la física sabiendo por qué y para qué hacen lo que hacen. Las deficiencias se extienden también a profesores de física y química de bachillerato, incluso cuando se enfrentan con ejemplos de este nivel (Martínez Torregrosa y López-Gay, 1992, 1997a; López-Gay et al., 2001a), provocando una impotencia reconocida por los propios docentes: de 103 profesores de bachillerato, participantes en cursos de formación en activo, encuestados mediante escalas de Likert, el $88 \%$ admite que «los propios profesores no dominan con seguridad suficiente el cálculo diferencial ante situaciones nuevas», y sólo un $22 \%$ se declara seguro de sus conocimientos sobre cuándo y por qué usar el cálculo diferencial en física.

Esta baja comprensión puede estar afectando notablemente a las expectativas y las actitudes. Los profesores tienen bajas expectativas de que sus alumnos de física de COU comprendan el uso del cálculo diferencial en la física y, por su parte, un $65 \%$ de los alumnos de COU (N $=108)$, un $77 \%$ de los alumnos de física de primer curso de carreras científico-técnicas $(\mathrm{N}=116)$ y un $64 \%$ de $2^{\circ}$ curso $(\mathrm{N}=63)$ admiten que «el profesor utiliza el cálculo diferencial porque lo necesita para el desarrollo del tema, pero él no espera que nosotros lo entendamos». En estas condiciones, resulta lógico que el uso del cálculo diferencial, en lugar de constituir una ayuda para avanzar en la comprensión física, sea percibido por los estudiantes como un obstáculo y una fuente de rechazo hacia la física, generador de inseguridad y ansiedad (Aghadiuno, 1992; Lavaly, 1990; Martin y Coleman, 1994; Monk, 1994).

Distintos trabajos coinciden en señalar que las deficiencias y dificultades encontradas en el uso del cálculo diferencial tienen su origen, principalmente, en una enseñanza inadecuada, caracterizada por un enfoque meramente algorítmico (Artigue y Viennot, 1987; Ferrini-Mundy y Gaudard, 1992; Ferrini-Mundy y Geuther, 1991; López, 1991; Nagy et al., 1991; Orton, 1983a y 1983b; Schneider, 1991; Thompson, 1994; Thompson y Thompson, 1994). A veces se justifica dicho enfoque algorítmico como un primer paso, adecuado para la iniciación. Sin embargo, algunos autores advierten que el adiestramiento intensivo en técnicas no favorece -antes bien, obstaculiza- la posterior comprensión conceptual (Ferrini-Mundy y Gaudard, 1992); tan sólo consigue, a lo sumo, una «comprensión instrumental», válida para reproducir rutinas manipulativas, pero insuficiente para resolver problemas de aplicación si no va acompañada de un conocimiento conceptual (White y Mitchelmore, 1996). El movimiento de reforma del cálculo iniciado en EEUU coincide en señalar el enfoque algo- rítmico como el causante de la situación crítica que atraviesa la enseñanza del cálculo en ese país, y propone un enfoque conceptual -preocupado por conseguir una verdadera comprensión de lo que se hace y por qué se hace- provocando con ello un interesante debate (Ferrini-Mundy y Gaudard, 1992; Jhonson, 1995; Kleinfeld, 1996; Knisley, 1997; Ostebee y Zorn, 1997; Swann, 1997; Tucker, 1997). Con este enfoque se han realizado distintos trabajos sobre los conceptos de derivada e integral o conceptos previos como el de límite o función (Azcárate, 1990; Bartle, 1996; Calvo, 1998; Confrey y Smith, 1994; Ferrini-Mundy y Geuther, 1991; López, 1991; Orton, 1983a, 1983b; Schneider, 1992; Thompson, 1994; Turégano, 1998).

Por nuestra parte, compartimos la necesidad de llevar a cabo un cambio de enfoque en el uso del cálculo diferencial en las clases de física, basado en una verdadera comprensión de lo que se hace y por qué se hace. A diferencia de la mayoría de los trabajos citados, no obstante, nuestra preocupación no se encuentra en el dominio puramente matemático sino en su aplicación en el contexto físico. Por esta razón, nuestro estudio se ha centrado en el concepto de diferencial, que es habitualmente relegado en la enseñanza de las matemáticas, mientras que, por el contrario, es muy utilizado en los razonamientos y matematización de situaciones físicas desde los últimos años del bachillerato, ya sea en los desarrollos teóricos o en la resolución de problemas, donde, desde los razonamientos iniciales, aparecen expresiones como: $\mathrm{d} e=v \cdot \mathrm{d} t, \mathrm{~d} p=F \cdot \mathrm{d} t, \mathrm{~d} W=F \cdot \mathrm{d} x, \mathrm{~d} V=E \cdot \mathrm{d} r$, $\mathrm{d} F=B \cdot I \cdot \operatorname{sen} \theta \cdot \mathrm{d} l, \mathrm{~d} N=-\lambda \cdot N \cdot \mathrm{d} t$, etc.

Para poder comprender las causas de las deficiencias en la enseñanza y aprendizaje habituales y elaborar propuestas que puedan superarlas, es necesario, previamente, clarificar conceptualmente el significado de todo lo que se hace cuando se usa el cálculo diferencial en las aplicaciones físicas. Hemos buscado realizar-dentro de un nivel que consideramos útil para los profesores de física de COU y primer curso universitario- esta clarificación de una forma problematizada: resaltando el problema que hace necesario recurrir al cálculo diferencial en la física, la estrategia general que se utiliza para resolverlo y el significado de los distintos conceptos y relaciones dentro de dicha estrategia. Para ello, hemos realizado un estudio histórico de la evolución del cálculo diferencial, en particular del concepto de diferencial, buscando los obstáculos que tuvieron que ser superados, las preguntas que necesitaron respuesta y los cambios que permitieron el avance hasta llegar a las concepciones actuales (Martínez Torregrosa et al., 1994). Lo que hemos aprendido de este estudio histórico y epistemológico nos ha permitido identificar con precisión en qué consiste una adecuada comprensión de la diferencial en la física, analizar e interpretar el origen de las deficiencias de la situación actual y planificar la enseñanza (Martínez Torregrosa y López-Gay, 1992, 1993, 1997a, 1997b; Gras Martí et al., 2001; López-Gay et al., 2001a, 2001b). En este artículo nos centramos en presentar la clarificación del concepto de diferencial y algunas de las ideas/obstáculo que hubo que superar hasta llegar a la concepción actual, concluyendo con una 
serie de criterios que serían indicadores de una comprensión adecuada de la diferencial.

\section{CONCEPCIONES HISTÓRICAS SOBRE LA DIFERENCIAL}

La invención del cálculo diferencial supuso un proceso de unificación de problemas, con independencia del campo específico al que perteneciesen, en dos grandes bloques: aquéllos que se resolvían mediante el método de derivación y los que se resolvían mediante el método de integración. Ambos métodos basaban su estrategia en el uso adecuado de las cantidades infinitamente pequeñas, lo que hizo que el nuevo cálculo fuese calificado de infinitesimal. Pero no sólo tenían en común la estrategia: Newton y Leibniz son considerados los creadores del cálculo diferencial por haber reconocido el carácter inverso de los dos tipos de problemas, reduciendo derivación e integración a operaciones inversas (Aleksandrov et al., 1956, p. 95; González Urbaneja, 1992, p. 68; Kline, 1972, p. 470).

El enorme éxito obtenido por el cálculo en los siglos XVII y XVIII para resolver un gran número de problemas no estuvo acompañado -contrariamente a la imagen de exactitud y rigor desde el principio que transmiten los textos de matemáticas- por una comprensión a prueba de dudas de lo que se hacía, lo que provocó una actitud mecánica que fue dejando al descubierto importantes deficiencias y contradicciones. En referencia a esa época, Eves (1981) afirma que «atraídos por la potente aplicabilidad del asunto, careciendo de una verdadera comprensión de los fundamentos sobre los que debe apoyarse, los matemáticos manipulaban los procesos analíticos de una manera casi ciega, a menudo guiados por una ingenua intuición de que lo que hacían debía ser válido» (p. 134).

Muchas de esas deficiencias afectaban directamente al significado y estatus de la diferencial. Desde el nacimiento del cálculo hasta el siglo $\mathrm{xx}$, el concepto de diferencial se debatió entre su identificación con las cantidades infinitamente pequeñas y su reducción a una expresión subordinada, carente de significado propio. Siguiendo la categorización usada por Alibert y otros (1987), hemos identificado dos concepciones históricas representativas de esta evolución: la diferencial de Leibniz y la diferencial de Cauchy. En este apartado se describirán brevemente las características de ambas concepciones, guiados por nuestro objetivo de entender mejor el uso habitual de la diferencial en la enseñanza de la física.

\section{La diferencial de Leibniz}

Las cantidades infinitamente pequeñas («cantidades divisibles evanescentes» según Newton, «cantidades incipientes "aún no formadas"» según Leibniz) constituyen la pieza fundamental para la creación del cálculo, pero también su punto más débil y el blanco de todas las críticas. Consideradas al principio de forma estática como cantidades fijas de valor más pequeño que cualquier número conocido pero nunca nulas, la concepción final adquirió un carácter dinámico: cantidades que podían hacerse tan pequeñas como se quisiera.

Leibniz y sus seguidores (hermanos Bernouilli, marqués de L'Hôpital, Euler...), cuya notación y lenguaje se impusieron en el cálculo diferencial, llamaban diferencial de una magnitud (dy) a la variación infinitesimal de esa magnitud (y) (su «momento», en palabras de Newton). Si dy hubiese podido tomar un valor macroscópico, no habría coincidido con $\Delta y$, pero, como sólo se le adjudicaban valores infinitamente pequeños, en ese rango se identificaba con $\Delta y$ sin cometer error alguno. Así, la diferencial de la posición (de), aunque en términos macroscópicos no correspondía a ningún desplazamiento, podía identificarse con el desplazamiento ocurrido en un intervalo de tiempo infinitamente pequeño $(\mathrm{d} t)$.

La diferencial ocupaba un lugar central en la estructura del cálculo y se utilizaba para sustituir el incremento para calcular la derivada (definida como el cociente de incrementos muy pequeños) y la integral (definida como una suma de infinitos incrementos muy pequeños). Los siguientes ejemplos ilustran el uso original de Newton y Leibniz de esas cantidades en sus cálculos y razonamientos, que ellos planteaban siempre en clave geométrica ${ }^{1}$ :

- Para calcular la derivada de la función $y=x^{2}$, consideraban que una variación infinitesimal $\mathrm{d} x$ produciría una variación también infinitesimal $\mathrm{d} y: y+\mathrm{d} y=(x+\mathrm{d} x)^{2}=$ $x^{2}+2 x \cdot \mathrm{d} x+\mathrm{d} x^{2}$; por tanto: $\mathrm{d} y=2 x \cdot \mathrm{d} x+\mathrm{d} x^{2}$. Después dividían ambos miembros por $\mathrm{d} x: d y / d x=2 x+d x$, y sólo en este momento despreciaban los sumandos infinitesimales, obteniendo: $\mathrm{d} y / \mathrm{d} x=2 x$.

- Para demostrar la relación inversa entre la derivación y el cálculo de áreas $A(x)$ bajo curvas $y(x)$, consideraban que una variación infinitesimal $\mathrm{d} x$ produciría una variación infinitesimal $\mathrm{d} A$, la cual podía aproximarse por el rectángulo de altura $y(x)$ y base $\mathrm{d} x$, resultando: $d A=y \cdot \mathrm{d} x$; dividiendo ambos miembros por $\mathrm{d} x$, obtenían la relación básica: $d A / \mathrm{d} x=y$.

Para ellos, por ejemplo, de sería un desplazamiento infinitesimal producido en el intervalo infinitesimal de tiempo d $t$ (que, aunque no era $\Delta e$, podía sustituirlo en ese intervalo tan pequeño) y la rapidez instantánea, el cociente entre estas cantidades infinitesimales.

Como puede apreciarse, el uso de los infinitesimales presentaba ciertas ventajas: se escribía como igualdad lo que sólo podía considerarse como aproximación si se utilizaban «incrementos finitos»-lo que resulta «doloroso» para un matemático actual, como señala Freudenthal (1973)-. Además, los términos que contenían estas cantidades podían despreciarse justo en el momento del razonamiento que se considerase oportuno, lo que resume el marqués de L'Hôpital mediante la ecuación: $y+\mathrm{d} y=y$. Pero, junto a estas ventajas, el uso de los 
infinitesimales generaba grandes dudas y fuertes críticas, que podemos resumir en las siguientes cuestiones:

- ¿Cómo puede justificarse la supresión de algunos términos? Decir simplemente que las cantidades suprimidas eran cero sin explicar por qué, al principio, no eran nulas y al final sí parecía violar el principio de identidad según el cual no existe estatuto intermedio entre la igualdad y la diferencia (aunque ésta sea muy pequeña) para dos entes matemáticos. George Berkeley era tajante al concluir: «[...] no pueden obtenerse proposiciones verdaderas de principios falsos» (citado por Rossi, 1997, p. 204).

- Otras veces se decía que no eran cero, pero sí despreciables frente a cantidades incomparablemente más grandes, en cuyo caso, ¿cómo puede obtenerse un resultado exacto despreciando términos que no son cero?

- ¿Cómo puede explicarse que la suma de infinitesimales, de cantidades despreciables, conduzca a un resultado finito?, se preguntaba el físico y geómetra holandés Nieuwentijdt (Kline, 1972, p. 509).

- ¿Con qué criterio se pasa de escribir una expresión sólo aproximada en términos de incrementos a otra exacta en términos de diferenciales? ¿Puede realizarse este paso para cualquier expresión? En el ejemplo citado más arriba, se aproxima el área de la curva por la de un rectángulo $(\Delta A \approx y \cdot \mathrm{d} x)$ e inmediatamente se escribe como igualdad en términos de diferenciales $(\mathrm{d} A=y \cdot \mathrm{d} x)$; pero no se trata de una deducción sino de una definición. No obstante, cuando se aplica esta definición para el cálculo del área de la superficie de un cuerpo geométrico concreto, aparecen distintas alternativas entre las que hay que escoger. Por ejemplo, cuando se desea hallar la expresión funcional exacta de la superficie de una esfera, puede estimarse $\Delta A$ mediante sumas de superficies cilíndricas infinitesimales o de superficies troncocónicas, lo que hace que no sea evidente cuál elegir como expresión para la diferencial (Artigue y Viennot, 1987), y se obtienen resultados distintos. ¿Cómo determinar la expresión diferencial? Menos evidente resulta en la mayoría de los problemas físicos, en los que son posibles muchas expresiones de partida que relacionan incrementos muy pequeños de forma aproximada. «La idea intuitiva de que la suma de infinitos "trocitos" infinitamente pequeños dará lugar al trozo grande deseado sin importar la "forma" de los trocitos fallaba en muchas ocasiones», conduciendo a resultados absurdos como los citados por Schneider (1991).

Newton y Leibniz fueron incapaces de responder con claridad a estas críticas y objeciones debido, en gran parte, a la falta de una definición precisa del concepto de límite. En los últimos trabajos de Newton existe un intento de abandonar el uso de los infinitesimales. «[...] En matemáticas no se deben despreciar ni los errores más diminutos», decía (citado por Kline, 1972, p. 480). Pero, todo era un intento formal para evitar contradicciones, ya que, como criticaba Berkeley, «al final es preciso volver a la idea de los incrementos evanescentes»
(Edwards, 1937, p. 294). Por su parte, Leibniz reconoce en alguna ocasión que él «no cree en magnitudes verdaderamente infinitas o verdaderamente infinitesimales» (Kline, 1972, p. 511); no obstante, defiende su uso por una cuestión meramente práctica, considerando los símbolos empleados «ficciones útiles para abreviar y hablar universalmente» (Edwards, 1937, p. 264). En su réplica a las críticas del físico Nieuwentijdt, el propio Leibniz afirma: «Se pueden utilizar estos entes últimos -esto es, cantidades infinitas e infinitamente pequeñas-como un instru-mento, en la misma forma en que los algebristas utilizaban las raíces imaginarias con gran provecho» (Kline, 1972, p. 509).

Este breve relato histórico muestra que el concepto de diferencial, identificado con un incremento infinitesimal, favoreció la construcción del cálculo y supuso un gran avance en la solución de problemas físicos. Sin embargo, ese mismo relato muestra también que esa definición de diferencial es insuficiente, no sólo por la falta de argumentos para explicar cómo y por qué funciona el cálculo, sino porque en muchas ocasiones conduce a resultados erróneos. En particular, la creencia errónea de que toda expresión aproximada del incremento puede considerarse exacta en intervalos infinitamente pequeños -es decir, cuando se transforma en una expresión diferencial- les impedía comprender por qué en unas ocasiones fallaba el algoritmo y en otras no, lo que generó inseguridad entre matemáticos y físicos de la época. El éxito obtenido por la aplicación del cálculo para resolver una gran cantidad de problemas, junto a la falta de comprensión y justificación de lo que se hacía, le imprimió un carácter de estrategia mecánica y repetitiva, más preocupada por el seguimiento fiel de algoritmos que por el significado, que -según las referencias citadas- todavía hoy perdura.

Los resultados presentados en otros trabajos (Artigue, 1986; Martínez Torregrosa y López-Gay, 1992, 1993, 1997a; López-Gay et al., 2001a) indican que esta concepción histórica y la actitud mecánica a la que conduce es dominante en la enseñanza habitual de la física. El siguiente fragmento de una entrevista realizada con un estudiante brillante de COU ilustra el uso de esta concepción:

Juan: «Cada vez que usamos diferenciales, mi profesor dice: "para estudiar esta curva vamos tomando rectas tan pequeñas como queramos..." [...] No lo tengo claro... La verdad, yo sé hacer integrales, pero no me he quedado muy bien con lo que son las diferenciales que aparecen; lo veo escrito pero no sé lo que son... y para qué voy a preguntar si me van a decir: "Estos son los trocitos chiquititos...”».

Aunque la diferencial de Leibniz, con sus dificultades y contradicciones, supuso un enorme avance para la comprensión y el estudio de la física, el mantenimiento de esta misma concepción (la diferencial de una función como cantidad infinitesimal que se aproxima al incremento infinitesimal de la función, pudiendo sustituirlo), en la enseñanza, tres siglos después, una vez que sabemos que es una concepción errónea, no parece ser lo más adecuado para promover la comprensión, la confianza y la autonomía en los estudiantes. 


\section{La diferencial de Cauchy}

Consciente de las imprecisiones y las ambigüedades del uso del infinito e infinitesimales, Lagrange convocó en 1784, en la Academia de Berlín, un concurso para reemplazar tales nociones sin perder simplicidad en los razonamientos. Ante la falta de respuestas satisfactorias, publicó su propia solución: una teoría de las funciones analíticas que liberaba el cálculo diferencial de los infinitamente pequeños y colocaba la noción de deriva$d a$ en un lugar preeminente ${ }^{2}$. Pero, como ya les había ocurrido a otros antes, no se trataba más que de un desarrollo teórico, pues en el momento de las aplicaciones físicas, como se refleja en su mecánica analítica, Lagrange recuperaba el uso de la diferencial y de los infinitamente pequeños (Laugwitz, 1997a).

La justificación rigurosa del cálculo llegó de la mano del matemático francés Cauchy, en la primera mitad del siglo XIX, quien, a partir de un mejor conocimiento del concepto de límite y del conjunto de los números reales, formuló una definición precisa de las cantidades infinitesimales, de la derivada y la integral. En cuanto a la diferencial, dejó de identificarse con un incremento infinitesimal, se vació de todo significado físico y pasó a ocupar un lugar marginal en la estructura del cálculo.

Cauchy define la cantidad infinitamente pequeña como una variable cuyo valor numérico decrece indefinidamente de manera que converge hacia el límite cero (Cauchy, 1821, pp. 26-27), lo que resulta ya suficiente para superar algunas de las objeciones que se habían formulado en los siglos anteriores. En concreto:

- Los infinitesimales no son cantidades muy pequeñas, sino variables $(x)$ o funciones $(f(x))$ que cumplen una propiedad: su límite, cuando $x$ tiende a cero, es cero. Esta propiedad no impone restricción alguna al valor numérico que puede tomar esa variable o función; por ejemplo: el incremento de cualquier función continua se ajusta a esta definición de infinitésimo, respecto a $\Delta x$, $\mathrm{y}$ dicho incremento puede tomar cualquier valor numérico.

- La aplicación del concepto de límite a una expresión produce un nuevo objeto matemático. Por ejemplo, en la función $y=x^{2}$ se puede calcular el cociente incremental: $\Delta y / \Delta x=2 x+\Delta x$, cuyo valor nunca será $2 x$, por muy pequeño que sea $\Delta x$. Sin embargo, en el momento en que se calcula el límite de esa expresión cuando $\Delta x$ tiende a cero, el nuevo objeto obtenido (que no es ningún cociente de incrementos, pues el límite de una sucesión no tiene por qué pertenecer a dicha sucesión) es igual a $2 x$. Por tanto, cuando en una expresión aparecen cantidades infinitesimales, dichas cantidades no son cero, pero sí lo son sus límites, justificando así no el que se desprecien sino que, en ese momento, se les iguale, a cero, exactamente a cero.

Conviene insistir en que estas ideas descansan sobre una adecuada comprensión del concepto de límite, lo cual no fue inmediato en tiempos de Cauchy ni se produce en la enseñanza actual de la física y las matemáticas (Cottrill,
1996; Lauten et al., 1994; Sánchez y Contreras, 1998; Schneider, 1992; Williams, 1991). El propio Cauchy dudaba sobre el significado de los infinitesimales: se refirió a veces al «valor» infinitamente pequeño de esas cantidades e incluso las acabó considerando como números muy pequeños, susceptibles de manipularse como entes independientes ${ }^{3}$. Esta confusión pudo llevar a Cauchy a prescindir de los infinitesimales en sus clases de análisis, decisión que le supuso enfrentarse con el físico Petit y el propio Consejo de Instrucción de la Escuela Politécnica, pues no entendían cómo podían omitirse los infinitesimales en las clases teóricas dada su utilidad para resolver problemas prácticos (Laugwitz, 1997b).

La definición de límite proporcionaba también una definición precisa de la derivada y la integral, y un procedimiento no ambiguo para calcularlas. La derivada se definió como «el límite de un cociente de incrementos»; la integral, que había sido reducida en la práctica a la operación inversa de la derivación después del enunciado del teorema fundamental, recuperó con Cauchy el importante papel que había jugado durante la primera mitad del siglo XVII y se definió como «el límite de una serie de sumas». Para el cálculo de ambas, se partía de una relación entre incrementos, aunque fuese aproximada, y después se calculaba el límite de un cociente o de una suma.

De esta forma, la diferencial no era ya necesaria para definir y calcular derivadas e integrales. Además, como el incremento de cualquier función continua obedece a la definición formal de infinitesimal, no tiene sentido utilizar el término diferencial para referirse al incremento (infinitesimal) de una función ${ }^{4}$. Si a esto se añade la sospecha acumulada a lo largo de los años sobre la diferencial y los infinitesimales de servir de base a tratamientos matemáticos poco rigurosos, el terreno resultaba claramente abonado para que la diferencial quedase relegada a un papel marginal en el nuevo marco teórico del cálculo.

Cauchy definió la diferencial como una expresión construida a partir de la derivada: $\mathrm{d} f=f^{\prime}(x) \cdot \mathrm{d} x$, siendo $\mathrm{d} x$ un incremento arbitrario (grande o pequeño) de la variable y pasó a convertirse así en un simple instrumento formal, necesario para justificar y abreviar ciertas demostraciones. Se desprendió, entonces, a la diferencial de la ambigüedad de los infinitamente pequeños, pero al mismo tiempo quedó desprovista de cualquier significado físico o intuitivo propio: simplemente era el producto de la derivada por el incremento de la variable independiente. Como afirma Freudenthal (1973, p. 550): «Diferenciales inútiles pueden ser despedidas de inmediato. Si $d y, d x$ aparecen sólo en la combinación $\mathrm{d} y / \mathrm{d} x$ o bajo el signo integral después del integrando, la pregunta sobre qué significan individualmente $\mathrm{d} x$, $\mathrm{d} y$ es equivalente a preguntarse qué significan las letras $l, o$, $g$, en $l o g$ ».

Esta concepción subordinada de diferencial, aunque satisface las exigencias de rigor matemático, no resulta satisfactoria en el contexto de las aplicaciones físicas, en las que las expresiones diferenciales -que carecían de 
significado físico propio para los seguidores de Cauchysiguieron constituyendo el punto de partida intuitivo para resolver la mayor parte de los problemas (como una aproximación del incremento, que coincide con él cuando es infinitesimal). Los matemáticos resolvían las ecuaciones diferenciales que planteaban los físicos sin ocuparse del significado de las mismas: simplemente dividían por $\mathrm{d} x$ y las convertían en ecuaciones en derivadas; a partir de ahí sólo la derivada y la integral tenían importancia. Los físicos, que seguían pensando en la diferencial de una manera intuitiva, muy cercana a la concepción de Leibniz, no podían aunar sentido físico y rigor. Por otro lado, si los matemáticos vaciaban de significado la diferencial, tampoco resolvían algunas de las cuestiones planteadas: ¿Con qué criterio se decide cuál es la expresión diferencial correspondiente a la situación física que se está estudiando?; por ejemplo, ¿qué argumento se utiliza para decidir cuál es la expresión diferencial que representa el proceso de absorción de una onda plana por un medio?

Pero no es sólo la ausencia de significado físico de las expresiones diferenciales el único inconveniente del nuevo análisis que nace con Cauchy, sino las dificultades que provoca el lenguaje puramente matemático alejado de la realidad física. Así, a pesar de disponer de una definición precisa de la derivada y la integral, es difícil reconocer la relación inversa entre ellas, algo que parecía intuitivo en la concepción de Leibniz. Más evidente aún es la dificultad para interpretar el significado físico de los conceptos y expresiones en las que aparecen; sirva de ejemplo el diálogo que encabeza un artículo del American Mathematical Monthly (citado por Cuenca, 1986):

Alumno: «El coche tiene una velocidad de 50 millas por hora, ¿qué quiere decir esto?»

Profesor: «[...] (Según Cauchy, $\lim _{\Delta t \rightarrow 0} \frac{e_{2}-e_{1}}{t_{2}-t_{1}}=50$

quiere decir que...) dado $\varepsilon>0$, existe un $\delta$ tal que si

$\left(t_{2}-t_{1}\right)<\delta$, entonces: $\frac{e_{2}-e_{1}}{t_{2}-t_{1}}-50<\varepsilon . »$

Esta respuesta tiene lugar cuando se renuncia a dar significado al concepto de diferencial, reduciendo el concepto de rapidez instantánea (o, en general, el de derivada) a su definición operativa como el cálculo de un límite.

Como puede apreciarse, el rigor adquirido por el cálculo en el siglo XIX, la «descolonización» y «vuelta a la cultura aborigen» (Aghadiuno, 1992), trajo consigo también un divorcio entre física y matemáticas. Ese divorcio se refleja aún hoy en la distinta perspectiva sobre la diferencial: en matemáticas es un instrumento formal que ocupa un papel marginal ${ }^{5}$; en física es un instrumento de aproximación, una cantidad muy pequeña, que ocupa un lugar central (Artigue, 1986; Artigue y Viennot, 1987).

Resulta evidente que la aportación de Cauchy no es suficiente para superar la sensación de inseguridad y la actitud mecánica cuando se usa el cálculo en las aplicaciones físicas, e incluso lo agrava al vaciar de significa- do un concepto tan importante para tales aplicaciones como es el de diferencial. Es necesario, pues, llevar a cabo una clarificación que consiga reconciliar, por un lado, la estrecha vinculación con las situaciones físicas de las expresiones diferenciales de Leibniz y Newton, y por otro el rigor y la precisión de su significado, saliendo al paso de la situación descrita por Freudenthal (1973, p. 553): «Es una situación imposible que el matemático enseñe unas matemáticas que no pueden ser aplicadas y el físico aplique unas matemáticas que no pueden ser enseñadas por el matemático.»

Este papel reconciliador lo ha jugado la concepción de diferencial introducida, en 1911, por el matemático francés Fréchet (Artigue, 1989, p. 34), para superar algunas deficiencias de la definición de Cauchy cuando se trataba de extender el análisis a funciones de varias e incluso infinitas variables (Alibert et al., 1987). Esta nueva definición (invención) de diferencial recupera un significado propio y preciso de gran interés físico y geométrico (como veremos) sin pérdida de rigor. No obstante, puesto que se produjo en un contexto muy alejado al que sería útil para profesores de bachillerato y de primeros cursos universitarios, nos hemos basado en ella para realizar una clarificación de la diferencial y de sus relaciones con la derivada y la integral de una forma problematizada (coherente con la naturaleza de hipótesis de los conceptos y con la intención de justificar cuándo, para qué y de qué manera se utiliza el cálculo diferencial en la física) y a un nivel que -según hemos probado en cursos de formación en activo en los que han participado más de cien profesores de COU- ha resultado provechoso para favorecer la transparencia conceptual sobre estas cuestiones (paso necesario, aunque no suficiente, para poder planificar su enseñanza adecuadamente).

\section{SIGNIFICADO Y UTILIDAD DE LA DIFE- RENCIAL EN LA FISICA (BASADO EN LA DEFINICION DE FRECHET)}

Como hemos señalado, no seguiremos el esquema habitual de los manuales de cálculo (definición, teorema, corolario...) para presentar la concepción de diferencial introducida hace menos de un siglo. Comenzaremos caracterizando las situaciones físicas cuya solución requiere el uso del cálculo diferencial, presentaremos una posible estrategia general para abordarlas y la pondremos en práctica resaltando cómo el desarrollo con éxito de dicha estrategia sólo es posible cuando los conceptos introducidos cumplen unas relaciones determinadas que delimitan con precisión sus significados.

\section{¿Cuál es el problema que hace necesaria la invención de la diferencial? ¿Qué estrategia se utiliza para resolverlo?}

El problema general que subyace en toda situación física que requiere el uso del cálculo diferencial es encontrar la expresión en forma de función que relaciona dos magni- 
tudes físicas: $x$ e $y$, obteniendo así la función incógnita $y(x)$. Si se conoce una condición inicial $y\left(x_{1}\right)=y_{1}$, el problema es equivalente a averiguar el $\Delta y$ producido por un cambio de variable desde $x_{1}$ hasta $x_{1}+\Delta x$ (Fig. 1). Por ejemplo, ¿cuál es la variación de rapidez de un móvil $(\Delta v)$ producida en un cierto intervalo de tiempo $(\Delta t)$ ? ¿Cuál es la variación de intensidad $(\Delta I)$ que experimenta una onda plana al atravesar un medio de espesor $\Delta x$ ? ¿Cuál es la variación de energía potencial de un muelle $(\Delta E)$ cuando se estira una distancia $\Delta x$ ? ¿Cuánto varía la presión atmosférica $(\Delta P)$ al ascender una altura $\Delta h$ ?

Figura 1

¿Cuál es el valor de $\Delta y$ correspondiente a $\Delta x$ ?

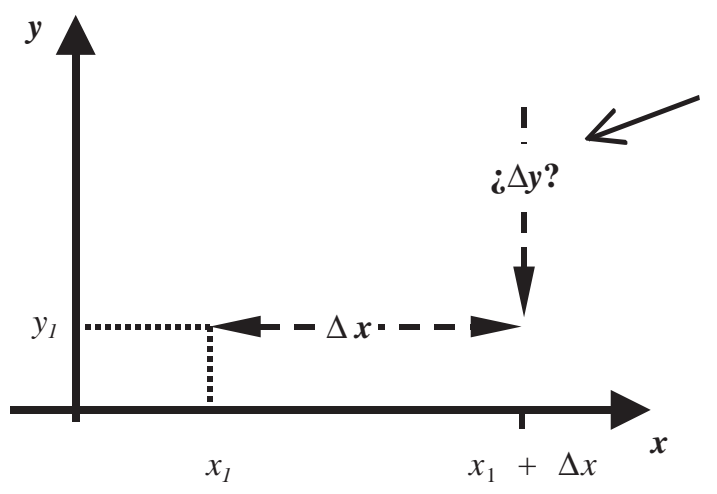

El punto de partida más sencillo es suponer que la gráfica es una recta, es decir, que la relación que liga $\Delta y$ con $\Delta x$ es lineal: $\Delta y=k \cdot \Delta x$ Cuando el conocimiento y el análisis físico de la situación, incluyendo la contrastación experimental, confirman esa dependencia, el problema está resuelto en su aspecto matemático, y sólo falta obtener el valor del parámetro $k$. Pero, en la mayoría de las situaciones, el análisis físico muestra precisamente que el comportamiento real no es de tipo lineal, es decir, que el coeficiente $k$ no es constante en el intervalo $\Delta x$, sino que varía con $x: k(x)$. ¿Qué valor de $k$ debe tomarse en estos casos? ¿Cómo avanzar en el objetivo de hallar $y=f(x)$, descartando el ensayo y error? Por ejemplo: si la aceleración tangencial varía con el tiempo, la fuerza depende del estiramiento o la densidad del aire varía con la altura, ¿cómo hallar el $\Delta v$ en un intervalo $\Delta t$, la variación de la energía potencial elástica al estirar $\Delta x$ o el cambio de presión, $\Delta P$, que se producirá al variar la altura $\Delta h$ ?

La estrategia para alcanzar nuestro objetivo en situaciones no lineales está basada en lo que Dieudonné (1960, p. 145) considera la idea fundamental del cálculo: la aproximación de funciones cualesquiera por medio de funciones lineales. En el siguiente cuadro se resumen los principales pasos de esa estrategia.
Cuadro I

Posible estrategia para hallar la relación funcional -una vez que sabemos que no es lineal- entre $\Delta y$ y $\Delta x$, a partir de funciones lineales.

1) Realizar una estimación del valor de $\Delta y$ a partir de $x$, y para un incremento $\Delta x$, suponiendo que la función tiene un comportamiento lineal a partir de $x_{1}$ y en todo el intervalo $\Delta x$ (ique puede ser tan grande como se quiera!). Representaremos esta estimación lineal por $\mathrm{d} y=k \cdot \Delta x\left(\right.$ donde $k$ es constante desde $x_{l}$ hasta $\left.x_{1}+\Delta x\right)$.

2) El error cometido al realizar esta estimación $(\Delta y-d y=\Delta y-k \cdot \Delta x)$ dependerá del valor de $\Delta x$ (en general, para un valor dado de $k$, el error será menor cuanto menor sea $\Delta x$ ) y del valor de $k$ (que puede ser cualquiera, es decir, es posible cualquier pendiente de la recta). Podemos, pues, mejorar la aproximación al $\Delta y$ dividiendo el intervalo $\Delta x$ en $N$ subintervalos, de valor: $\Delta x_{i}=x_{i+1}-x_{i}$, calculando una estimación lineal del $\Delta y_{i}$ correspondiente $\left(\mathrm{d} y_{i}=k_{i} \cdot \Delta x_{i}\right)\left(k_{i}\right.$ se mantiene constante entre $x_{i}$ y $x_{i}+\Delta x_{i}$ ), y sumando las estimaciones lineales parciales para obtener una estimación total: $\Delta y \approx \sum d y_{i}$. Si el error cometido en cada subintervalo es $\varepsilon_{\mathrm{i}}=\Delta y_{i}-\mathrm{d} y_{i}=\Delta y_{i}-k\left(x_{i}\right) \cdot \Delta x_{i}$, tendremos:

$$
\begin{aligned}
\Delta y & =\sum_{i=1}^{N} \Delta y_{i}=\sum_{i=1}^{N}\left(d y_{i}+\varepsilon_{i}\right)=\sum_{i=1}^{N} d y_{i}+(\text { error total }) \\
& =\sum_{i=1}^{N} k\left(x_{i}\right) \cdot \Delta x_{i}+(\text { error total })
\end{aligned}
$$

La calidad de esa aproximación aumenta disminuyendo el valor de cada $\Delta x$, es decir, aumentando el valor de $N$. Para poder realizar la estimación para cualquier $N$, hemos de disponer de un valor de $k$ para cada $x$, pasando así de un conjunto discreto de $k_{\text {. }}$ a una función: $k(x)$. La calidad de la aproximación dependerá del valor de $N$ y de la $k(x)$ elegida.

3) Cambiando el valor de $N$ se obtiene una serie de estimaciones totales del $\Delta y$, y una serie de errores totales. El límite de la serie de estimaciones, cuando $N \rightarrow \infty$, será exactamente $\Delta y$ si, y sólo si, el límite de la serie de errores totales, cuando $N \rightarrow \infty$, es cero. Es decir:

$$
\begin{aligned}
& \lim _{\mathrm{N} \rightarrow \infty} \sum_{i=1}^{N} d y_{i}=\lim _{\mathrm{N} \rightarrow \infty} k\left(x_{i}\right) \cdot \Delta x_{i}=\Delta y \\
& \text { si, y sólo si: } \lim =\sum_{\mathrm{N} \rightarrow \infty} \varepsilon_{i}=0
\end{aligned}
$$

Esto no ocurrirá necesariamente para cualquier función $k(x)$, aunque $\Delta x \rightarrow 0$ (éste era el error que llevaba a resultados absurdos en la concepción de Leibniz). Pero, si podemos encontrar la función $k(x)$ que hace que eso ocurra, el problema quedará resuelto: habremos obtenido la función incógnita $y=f(x)$ a partir del límite de una suma de estimaciones lineales de pendiente $k(x)$.

Vamos a poner en práctica con detalle esta estrategia para ver si existe una solución general al problema de encontrar la relación entre $\Delta y$ e $\Delta x$ o, si se conoce una condición inicial, la función incógnita.

1) Aunque sabemos que $\Delta y$ no es lineal respecto a $\Delta x$, podemos realizar una estimación de su valor suponiendo que la función tiene un comportamiento lineal (es una recta), es decir, su pendiente es constante en el intervalo (¡que no es infinitesimal!) $\Delta x: \mathrm{d} y=k \cdot \Delta x$. Así pues, estamos seguros de que dy no coincide con $\Delta y$ : representa lo que variaría la función desconocida en un $\Delta x$, a partir de $x_{1}$, si lo hiciera linealmente con una pendiente $k$ (Fig. 2). Para dar simetría a esa expresión, suele escribirse $\mathrm{d} y=k \cdot \mathrm{d} x$, pues $\mathrm{d} x=\Delta x$ (la función $y=x$ es lineal, con $k=1$ ). No obstante, existen infinitos valores posibles para la pendiente $k$, es decir, infinitas estimaciones lineales (Fig. 3). 
Figura 2

dy es una estimación del $\Delta y$, lineal respecto al $\Delta x$.

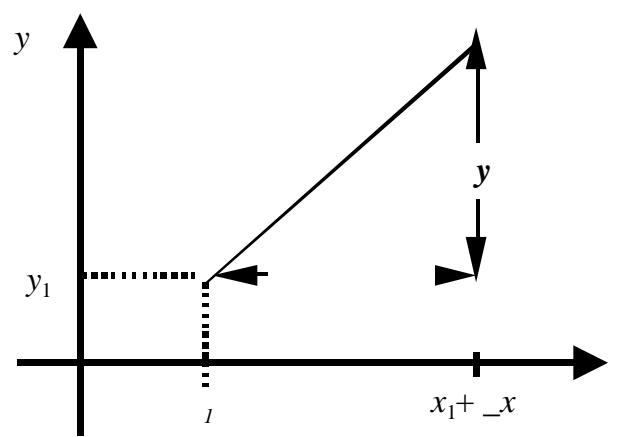

¿Cualquiera de ellas es válida o sólo hay una que permita resolver el problema (hallar el valor exacto de $\Delta y$ )?

2) En general, cuanto menor sea el $\Delta x$, el error cometido mediante la estimación diferencial es menor, pues la suposición $k$ se mantiene constante se acerca más al comportamiento real. Por ello, una mejora de la estimación consiste en dividir el intervalo $\Delta x$ en $N$ subintervalos, calcular la estimación $d y_{i}$ correspondiente a cada subintervalo y después sumar. Como esa partición del intervalo completo puede ser cualquiera, no sólo nos interesa conocer el valor de la diferencial a partir de $x_{l} \mathrm{y}$ para todo el intervalo, sino a partir de cualquier $x$ y para todo $\Delta x$. La diferencial es, por tanto, una función de dos variables $(x, d x)$ cuya expresión tendrá la forma $\mathrm{d} y=$ $k(x) \cdot \mathrm{d} x$.

Figura 4

Representación gráfica, en el sistema de coordenadas $y$ - $x$, del intento de mejora de la estimación del $\Delta y$ mediante el cálculo de dy consecutivas. $k\left(x_{i}\right)$ es la pendiente del segmento más grueso $(\mathrm{d} y / \Delta x)$ La suma de todos los segmentos verticales es una estimación del $\Delta y$.
Figura 3

Para un valor dado $x$, existen infinitas funciones lineales que permiten estimar el valor de $\Delta y$ para un $\Delta x$.

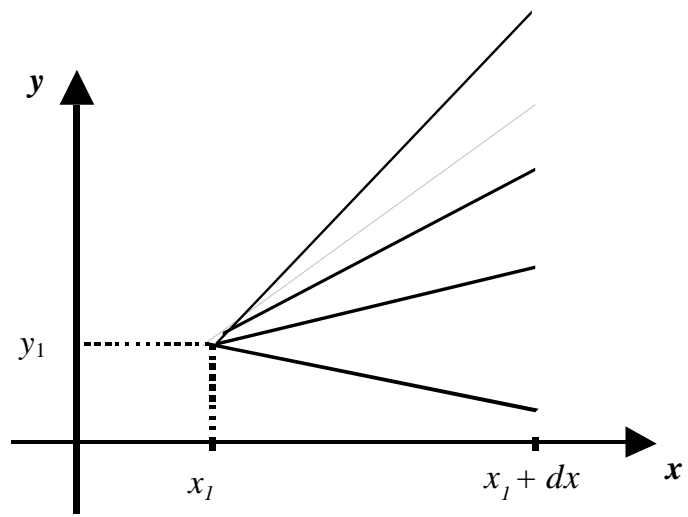

La mejora de la estimación que se ha realizado se expresa:

$$
\Delta y \approx \sum_{i=1}^{N} d y_{i}=\sum_{i=1}^{N} k_{i} \cdot d x_{i} \quad(\text { Fig. } 4 \text { y } 5) .
$$

Esa suma de estimaciones lineales no es igual a $\Delta y$ : se comete un error total que es la suma de los errores parciales. Llamando $\varepsilon_{\mathrm{i}}$ a cada error parcial $^{6}$ cometido $\left(\Delta y_{i}-\mathrm{d} y_{i}\right)$, y error total, a la suma de esos errores parciales, entonces:

$\Delta y=\sum_{i=1}^{N}\left(d y_{i}+\varepsilon_{i}\right)=\sum_{i=1}^{N} d y_{i}+($ error total $)$

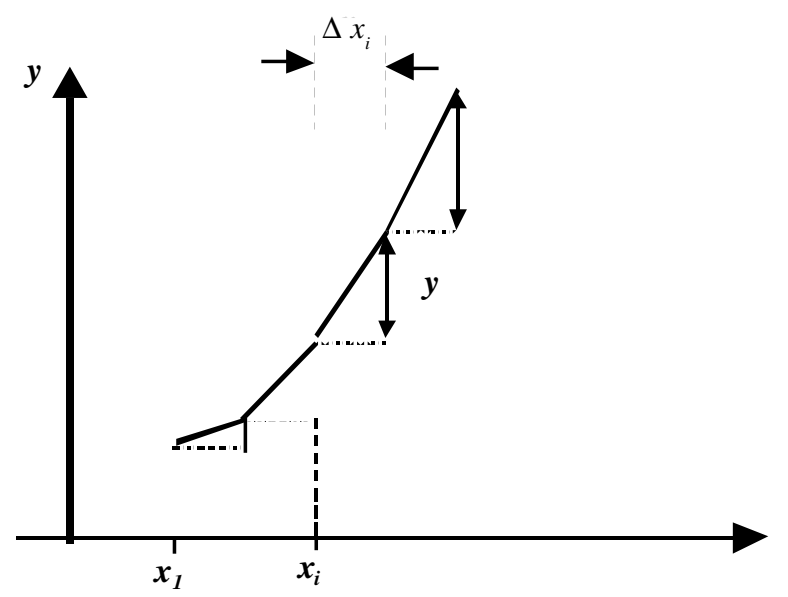

Figura 5

Representación, en el sistema de coordenadas $k$-x, del intento de mejora de la estimación del $\Delta y$ mediante el cálculo de dy consecutivas. $\mathrm{d} y=k(x) \cdot \Delta x$ es el área del rectángulo rayado. La suma del área de todos los rectángulos es una estimación del $\Delta y$.

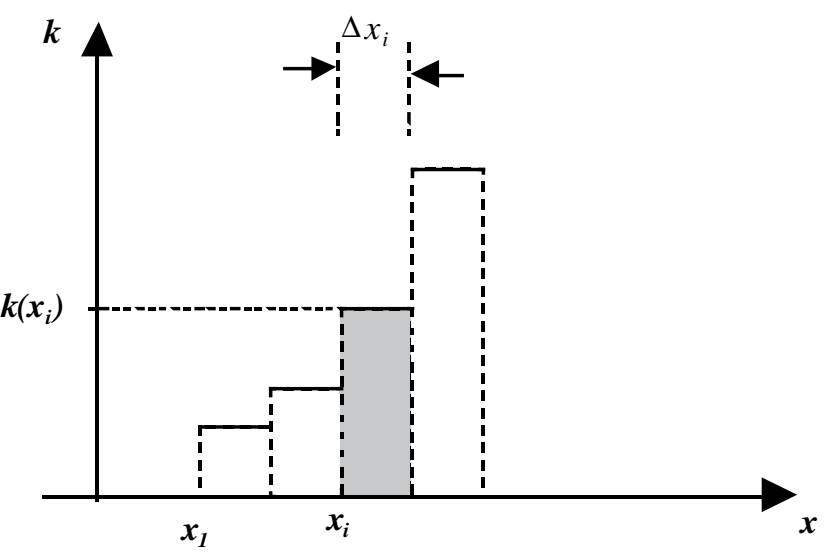


Cada error parcial $\varepsilon$. depende del valor de $\Delta x$. (determinado por el número $N$ de subintervalos) y del correspondiente $k_{i}$ (determinado por la función $k(x)$ elegida). Por tanto, el error total depende de $N$ y $k(x)$.

Para una función $k(x)$ determinada, dando distintos valores a $N$ se obtiene una serie de estimaciones globales y su correspondiente serie de errores totales. Ninguno de los términos de esta última serie será nulo, pues $\varepsilon_{\mathrm{j}}$ es siempre distinto de cero por muy grande que sea $N$, ya que el comportamiento seguirá siendo no lineal por muy pequeño que sea el intervalo $\Delta x_{i}$. Tampoco tiene que ser necesariamente cero el límite de esa serie cuando $N$ tiende a infinito, aunque -ahora sí- existe la posibilidad de que así ocurra ${ }^{7}$, dependiendo de qué comportamiento sea dominante: el del número de sumandos $(N)$, que se hace cada vez mayor, o el de cada sumando $\left(\varepsilon_{i}\right)$, que se hace cada vez menor.

Aclarada esta posibilidad, el límite de la serie de sumas de estimaciones: $\lim _{N \rightarrow \infty} \sum_{i=1}^{N} d y_{i}$ (que recibe el nombre de $i n$ tegral y se representa por $\int_{x_{1}}^{x_{1}+\Delta x} d y$ ) será exactamente $\Delta y$ si, y sólo si, el límite de la serie correspondiente de errores totales es cero. Como el límite de esa serie depende de la función $k(x)$ elegida, el problema quedará resuelto si sabemos encontrar la función $k(x)$, que hace que el límite de la serie de errores totales sea cero.

3) Sea cual sea la estimación lineal de partida, siempre se cumplirá que el límite de cada error parcial $\left(\Delta y_{i}-\mathrm{d} y_{i}\right)$ será cero. Sin embargo, la condición que buscamos es más exigente: que sea cero el límite del error total. Ello nos permitirá seleccionar una de entre todas las estimaciones lineales posibles. Lo que garantiza que el límite del error total sea cero es que el límite de $N$ veces cualquiera de los errores parciales sea cero. Es decir:

$$
\int_{x_{1}}^{x_{1}+\Delta x} d y=\Delta y \text { si, y sólo si: } \lim _{\mathrm{N} \rightarrow \infty} \mathrm{N} \cdot(\Delta y-d y)=0 \quad \forall x
$$

Como $N$ y d $x$ son inversamente proporcionales (si los subintervalos son iguales: $N=\Delta x / \mathrm{d} x$ ), entonces:

$$
\int_{x_{1}}^{x_{1}+\Delta x} d y=\Delta y \text { si, } y \text { sólo si: } \lim _{d x \rightarrow 0} \frac{\Delta y-d y}{d x}=0 \quad \forall x
$$

El límite de una resta es la resta de los límites, y además el cociente $\mathrm{d} y / \mathrm{d} x$ es constante para cada $x$, pues debe recordarse que dy es una estimación lineal respecto a $\mathrm{d} x$. Por tanto:

$$
\int_{x_{1}}^{x_{1}+\Delta x} d y=\Delta y \text { si, } y \text { sólo si: } \lim _{d x \rightarrow 0}\left(\frac{\Delta y}{d x}\right)-\frac{d y}{d x}=0 \quad \forall x
$$

Como d $x$ es el tamaño del subintervalo, el cambio de variable, el único límite que aparece en esa expresión es la definición de derivada:

$$
\int_{x_{1}}^{x_{1}+\Delta x} d y=\Delta y \text { si, } y \text { sólo si: } y^{\prime}=\frac{d y}{d x} \forall x
$$

Conclusión: Para que una estimación lineal: $\Delta y \approx k(x) \cdot \mathrm{d} x$ sea la diferencial, debe cumplir que su pendiente $k(x)$ coincida con la función derivada: $y$ ' $(x)$, lo que permitirá llegar al resultado exacto aplicando la estrategia del cálculo.

En la búsqueda de solución al problema, ha quedado establecida con claridad la relación inversa entre integración y derivación, lo que constituye el teorema fundamental. Para resolver una integral del tipo $\int k(x) \cdot \mathrm{d} x$, basta identificar el integrando con una diferencial: $k(x) \cdot \mathrm{d} x=\mathrm{d} y$ :

$$
\int_{x=a}^{x=b} k(x) \cdot d x=\int_{a}^{b} d y=\text { si, } y \text { sólo } \Delta y \text { si: } y^{\prime}=\frac{d y}{d x}=k(x) \forall x
$$

\section{¿Qué es la diferencial?}

Como vemos, es dentro de esta estrategia global del cálculo donde la diferencial, la derivada y la integral definida, así como las relaciones entre ellos, adquieren un significado claro y justificado. A modo de resumen, podemos formular ahora una definición precisa de diferencial de una magnitud (y) respecto de otra $(x)$ : es la única función lineal del incremento $(\mathrm{d} y=k(x) \cdot \mathrm{d} x)$, la única estimación lineal del $\Delta y$, que permite obtener la relación exacta entre $\Delta y$ y $\Delta x$ vía integral, y para ello su pendiente, $k(x)$, debe coincidir con la función derivada: $y^{8}$.

Algunas preguntas básicas relacionadas con el uso del cálculo diferencial en la fisica ( $i$ Tienen realmente un significado físico las expresiones diferenciales? ¿Es la diferencial una cantidad infinitesimal? ¿Es la diferencial una aproximación? ¿Es la derivada un verdadero cociente de diferenciales que pueden, por tanto, despejarse? ¿Cuál es el papel del símbolo diferencial que aparece dentro de las integrales? ¿Por qué las integrales (definidas) se calculan mediante reglas inversas a las de derivación?...) adquieren una respuesta sencilla después de la definición de diferencial que se ha presentado. Por ejemplo, de a partir de un instante $t$ y para un intervalo $\mathrm{d} t$ representa lo que variaría la posición en ese intervalo si lo hiciera uniformemente, es decir, con rapidez constante $^{9}$. Al estudiar la dependencia de la presión atmosférica con la altura, si escribimos una expresión para $\mathrm{d} P$, para una altura $h$, y un intervalo $\Delta h$ (o d $h$ ) significa lo que variaría $P$, desde $h$ hasta $h+\Delta h$, si lo hiciera linealmente respecto a $\Delta h$, es decir, con una rapidez de cambio con la altura constante a lo largo de todo el intervalo. $\mathrm{d} P$, por tanto, puede tomar cualquier valor, dependiendo de $h$ y de $\mathrm{d} h$.

El significado gráfico de la integral, en el sistema de coordenadas $k$ - $x$, también queda aclarado (Fig. 6). El área bajo la curva no es la suma de áreas de rectángulos, sino un objeto nuevo: el límite de la serie de sumas de rectángulos.

Al poner de manifiesto la debilidad de considerar la diferencial como una aproximación infinitesimal del incremento, no renunciamos, como era el caso de 
Figura 6

El valor del área de la figura rayada, en el sistema de coordenadas $k-x$, será $y(b)-y(a)$, si y sólo si $k(x)=y^{\prime}(x)$

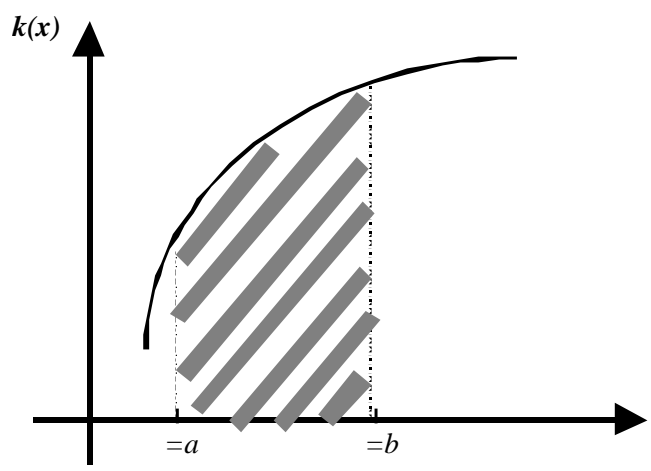

Cauchy, a dar significado físico a las expresiones diferenciales, reduciéndolas a una simple cuestión de lenguaje, a un instrumento formal. Por el contrario, la clarificación que hemos realizado, según una lógica problematizada, nos ha llevado a mantener la idea intuitiva de aproximación, definiendo la diferencial como una estimación lineal del incremento, pero eliminando la condición superflua de infinitesimal. Sólo se añade una condición: que la pendiente de la estimación coincida con la derivada, precisamente para garantizar que se trata de la única estimación que hace que el error total acumulado sea cero cuando se calcula la integral.

\section{Naturaleza hipotética de la diferencial en física}

Aunque hemos visto que, si conocemos la función diferencial, podemos hallar el $\Delta y$ buscado, vía integral, la situación habitual en física es que se desconoce tanto la función $y=f(x)$ como su función derivada $y^{\prime}=g(x)$. En ese caso, es necesario avanzar a título de hipótesis, apoyados en el análisis y el conocimiento físico de la situación, una estimación lineal del $\Delta y$ respecto al $\Delta x$, suponer que es la estimación diferencial y obtener el resultado global al que conduce (la función incógnita) que sí es contrastable (experimentalmente o por su coherencia con el cuerpo de conocimientos en que se inserta el problema). Así, por ejemplo, si queremos hallar cómo varía la intensidad, $I$, de una onda plana al atravesar un medio, es decir, $I(x)$, siendo $x$ la distancia atravesada por la onda en el interior del medio, desconocemos $I(x)$ y, por supuesto, también su derivada. Podemos, no obstante, formular hipótesis razonables sobre los factores que influirán en el $\Delta I$ producido al atravesar una distancia $\Delta x$, y concretarlas en una estimación lineal del $\Delta I$. En este caso, por ejemplo, cabe esperar que $\Delta I$ dependa de la naturaleza del medio atravesado, de $\Delta x$ y del valor de la intensidad (a mayor intensidad, mayor absorción por el medio, en un mismo $\Delta x$ ), pero no podemos escribir $\Delta I=-\alpha \cdot I \cdot \Delta x$, pues sabemos que $I$ varía con $x$ (es decir, que $\Delta I$ no es lineal respecto a $\Delta x: \frac{\Delta I}{\Delta x} \neq c t e$. ). Pero una estimación lineal del $\Delta I$, al pasar de $x$ a $x+\Delta x$, podría ser $\mathrm{d} I=-$ $\alpha \cdot I(x) \cdot \mathrm{d} x$, que representa lo que valdría $\Delta I$, a partir de $x$ $\mathrm{y}$ en un $d x$ (que es exactamente lo mismo que $\Delta x$ y no tiene por qué ser pequeño) si la variación de $I$ se produjera uniformemente, siempre al mismo ritmo, desde el comienzo del intervalo. También serían igualmente posibles expresiones como $\mathrm{d} I=-\alpha \cdot I^{2}(x) \cdot \mathrm{d} x, \mathrm{~d} I=-\alpha \cdot I^{1 / 2}(x) \cdot \mathrm{d} x$ o $\mathrm{d} I=-\alpha \cdot \mathrm{d} x / x$.

La expresión elegida conducirá, vía integral, a una expresión funcional de $\Delta I \mathrm{o}$-si se conoce una condición de contorno- de $I=f(x)$, que puede ser sometida a contrastación, lo que permite aceptarla o no y, en consecuencia, aceptar o no la expresión diferencial seleccionada. Lamentablemente, en los libros de texto no aparecen estas consideraciones sobre la naturaleza hipotética de la diferencial, transmitiendo una falsa e incomprensible sensación de seguridad a priori. No es infrecuente, incluso, encontrar expresiones del tipo «se comprueba experimentalmente que $\mathrm{d} I=\ldots \gg$

No obstante, sí que existen situaciones en que es posible asegurar desde el principio la expresión diferencial adecuada. Esto ocurre cuando, por la definición de la magnitud, conocemos con certeza la relación entre $\Delta y$ e $\Delta x$ cuando la situación física es tal que ésta es lineal $(\Delta y=k \cdot \Delta x)$ pero queremos encontrar dicha relación en una situación en que $k$ varía con $x$. Tendremos que recurrir a la estrategia general del cálculo, pero, en este caso, la mejor estimación lineal de $\Delta y$, la diferencial, será obviamente: $\mathrm{d} y=k(x) \cdot \mathrm{d} x$. Por ejemplo, sabemos que la variación de energía total de un sistema físico que se produce debido al trabajo exterior realizado por una fuerza constante cuyo punto de aplicación se desplaza una distancia $\Delta x$, es $\Delta E=F \cdot \Delta x$, pero si la fuerza varía con la distancia, de la forma $F(x)$, ya no conocemos la función que relaciona la variación de energía con el desplazamiento. Para hallar $\Delta E$ será necesario recurrir a la estrategia general del cálculo, y la estimación diferencial será: $\mathrm{d} E=F(x) \cdot \mathrm{d} x$.

\section{INDICADORESDE UNA COMPRENSIÓNADE- CUADA DE LA DIFERENCIAL EN EL ULTI- MO CURSO DE BACHILLERATO Y PRIMER CURSO DE ESCUELAS Y FACULTADES}

El objetivo de esta clarificación, no lo olvidemos, era proporcionar una guía (basada en la transparencia conceptual que aportan los conocimientos actuales del cálculo y en lo aprendido de la historia de su evolución) para analizar y comprender la situación actual del uso del cálculo diferencial en la física y buscar propuestas de mejora. En concreto, lo hecho hasta aquí permite avanzar de un modo fundado cuáles serían los indicadores de una comprensión adecuada del concepto de diferencial en el campo de la física en los niveles citados y valorar en qué medida, en libros de texto, profesores y alumnos que han superado dichos cursos están o no presentes y dónde pueden existir deficiencias. 
1) Saber cuándo y por qué se hace necesario su uso, es decir, conocer cuál es el problema que hace insuficiente el cálculo ordinario; en concreto, saber que es necesario recurrir a la diferencial cuando queremos hallar el $\Delta y$ producido en un $\Delta x$, y la relación entre $\Delta y$ e $\Delta x$ no es lineal $\left(\frac{\Delta I}{\Delta x} \neq\right.$ cte. $)$.

2) Conocer la estrategia que utiliza el cálculo para resolver ese problema y comprender el sentido de los distintos pasos que se recorren; en concreto:

- Saber explicar con precisión y sentido físico el significado de las expresiones diferenciales, reconocer sin ambigüedad que la diferencial puede tomar valores numéricos e interpretar el significado de los mismos.

- Conocer y justificar la relación que existe entre la diferencial $(d y)$ y la derivada $\left(y^{\prime}\right): y^{\prime}=\frac{d y}{d x}, y$ aceptar sin ambigüedad los razonamientos en los que se utiliza esa relación.

- Conocer el significado de la integral y saber justificar el denominado teorema fundamental, es decir, por qué la integral definida requiere el cálculo de antiderivadas o funciones primitivas.

\section{NOTAS}

${ }^{1}$ El análisis del siglo XVII tenía como objeto de estudio las curvas geométricas, y fue Euler, en el siglo XVIII, el encargado de separar análisis y geometría, dando una definición precisa de función y convirtiendo este concepto en el objeto de estudio del análisis.

${ }^{2}$ Es significativo su cambio de notación para referirse a la derivada, sustituyendo la expresión d $f / \mathrm{d} x$ por esta otra: $f^{\prime}$. Este mismo cambio se produce al pasar de la asignatura de física a la de matemáticas.

${ }^{3}$ Laugwitz (1997b) considera a Cauchy el precursor del conjunto de los números hiperreales, definidos por Robinson ciento cuarenta años más tarde en el desarrollo del análisis no estándar (NSA).

${ }^{4}$ Tampoco tiene sentido definir la diferencial como el límite del incremento, como hace textualmente algún libro de física general escribiendo: $d s=\lim _{\Delta s \rightarrow 0} \Delta s$. Es evidente que, entonces, la diferencial sería siempre cero.

${ }^{5}$ Este divorcio se manifiesta incluso en trabajos expresamente dirigidos a la mejora de la enseñanza del cálculo. En concreto, en uno de ellos (Orton, 1983b), realizado claramente desde la perspectiva matemática, se advierte que los símbolos $d x$, dy no tienen significado por sí solos, y que sólo significan algo cuando están juntos en la forma dy/dx, o cuando se usa dx en la integración.

${ }^{6} \mathrm{El}$ signo de cada $\varepsilon_{i}$ puede ser distinto, según que la estimación realizada difiera por exceso o por defecto en cada subintervalo. En lo que sigue, se considera que los $\varepsilon_{i}$ son todos positivos para evitar trabajar con valores absolutos y facilitar la lectura del texto.
- Utilizar con sentido esa estrategia en situaciones y problemas en los que se domine el contenido físico de los mismos.

3) Ser consciente de la naturaleza hipotética, tentativa, en casi todas las situaciones físicas, de la expresión diferencial de partida, y saber que la validez de esa hipótesis no puede ser contrastada directamente sino a través del resultado al que conduce.

4) Valorar positivamente el papel de la diferencial en el aprendizaje de la física. Este componente axiológico debería ser una consecuencia natural cuando se comprende el papel crucial que juega la diferencial en el tratamiento de situaciones físicas de interés.

Los resultados obtenidos al analizar libros de texto y la presentación didáctica habitual que realizan los profesores, que serán presentados en otro lugar, confirman de un modo rotundo la presencia generalizada de deficiencias en los indicadores mencionados. Al mismo tiempo, disponemos de resultados prometedores -aunque es un trabajo todavía en curso- sobre la mejora general de los mismos con la incorporación de la nueva orientación a la enseñanza desde el primer momento en que se plantea la necesidad de utilizar el cálculo diferencial (López-Gay et al., 2001b).

${ }^{7}$ A fin de salir al paso de algunas dificultades para aceptar esta posibilidad, conviene advertir de ciertos errores relacionados con el concepto de límite; en concreto, no se trata de un proceso sin fin sino de que el límite es un valor concreto, que además no tiene por qué coincidir con ninguno de los términos de la serie o de la sucesión, sino que constituye un objeto mental nuevo (Cottrill, 1996; Lauten et al., 1994; Sánchez y Contreras, 1998; Schneider, 1992; Williams, 1991).

${ }^{8}$ Esta condición equivale a exigir que la diferencial $(\mathrm{d} y)$ no difiera del incremento $(\Delta y)$ más que en un infinitamente pequeño con relación a $\Delta x$. Por tanto, lo que debe ser infinitamente pequeño, respecto a $\Delta x$, no es ni $\Delta y$ ni d $y$, sino su diferencia $(\Delta y-\mathrm{d} y)$. Para salir al paso de incorrectas interpretaciones, conviene advertir que esa condición no significa que $(\Delta y-\mathrm{d} y)$ sea siempre un número muy pequeño, y mucho menos que $\Delta y$ o d $y$ lo sean. El significado correcto de la expresión: « $(\Delta y-\mathrm{d} y)$ es infinitamente pequeño con relación a $\Delta x »$ es que $(\Delta y-\mathrm{d} y)$ tiende a cero más rápidamente que $\Delta x$, es decir, que el límite de $(\Delta y-\mathrm{d} y) / \Delta x$ es cero cuando $\Delta x$ tiende a cero.

${ }^{9}$ Con esta concepción del significado de de, se puede superar el pensar en la rapidez instantánea como una rapidez media en un intervalo de tiempo infinitesimal, como era el caso de Leibniz. La rapidez en un instante de un móvil es la rapidez que tendría el móvil a partir de dicho instante si se moviera uniformemente. La rapidez instantánea será, pues, $v=\frac{\mathrm{de}}{\mathrm{dt}}$. Otra cuestión es cómo se halla operativamente el valor de dicho cociente. 


\section{REFERENCIAS BIBLIOGRÁFICAS}

AGHADIUNO, M.C.K.(1992). Mathematics: history, philosophy and applications to science. International Journal for Mathematical Education in Science and Technology, 23(5), pp. 683-690.

ALEKSANDROV, A.D. et al. (1956). La matemática: su contenido, métodos y significado. Madrid: Alianza Universidad, 7a. ed., 1985

ALIBERT, D. et al. (1987) Le thème «Differentielles» un exemple de coopération maths-physique dans la recherche. Actes du Colloque du GRECO Didactique et Acquisition des Connaissances Scientifiques, Sêvres, pp. 7-45. Grenoble: La Pensée Sauvage,

ARTIGUE, M.(1986). The notion of differential for undergraduate students in Science. Proceedings of the XAnnual conference of the International Group for the Psychology of Mathematics Education, pp. 229-234. Londres.

ARTIGUE, M. (1989). Le passage de la différentielle totale à la notion d'application linéaire tangente, en Procedures différentielles dans les enseignements de mathematiques et de physique au niveau du premier cycle universitaire (Annexe I). IREM et LDPES: Université Paris 7.

ARTIGUE, M. y VIENNOT, L. (1987). Some aspects of students' conceptions and difficulties about differentials. Second International Seminar Misconceptions and Educational Strategies in Science and Mathematics, Cornell (vol. III). Ithaca: Cornell University.

AZCÁRATE, C. (1990). «La velocidad: introducción al concepto de derivada». Tesis doctoral. Universidad Autónoma de Barcelona. Reseña en Enseñanza de las Ciencias, 9(2), pp. 201-202.

BARTLE, R. (1996). Return to the Riemann Integral. The American Mathematical Monthly, 103(8), pp. 625-632.

CALVO, C. (1998). «Bases para una propuesta didáctica sobre integrales». Tesis de maestría. Reseña en Enseñanza de las Ciencias, 16(1), pp. 194-195.

CAUCHY, A.L. (1821). Cours d'Analyse de L'École Royale Polytechnique. Edición facsímil de la primera edición. Sevilla: Sociedad Andaluza de Educación Matemática Thales, 1998.

CONFREY, J. y SMITH, E. (1994). Exponential functions, rates of change, and the multiplicative unit. Educational Studies in Mathematics, 26, pp. 135-164.

COTTRILL, J. et al. (1996). Understanding the Limit Concept: Beginning with a Coordinated Process Scheme. Journal of Mathematical Behavior, 15, pp. 167-182.

CUENCA, J.A. (1986). Iniciación al análisis no estándar (1a., 2a. y 3a.parte). Revista de la Sociedad Andaluza de Profesores de Matemáticas «Thales», 5, pp. 34-48; 6, pp. 42-51; 7, pp. 27-43.

DIEUDONNÉ, J. (1960). Fundamentos de análisis moderno. Barcelona: Reverté, 1974

EDWARDS, C.H. (1937). The Historical Development of the Calculus. Nueva York: Springer-Verlag, 1979.

EVES, H. (1981). Great moments in Mathematics (After 1690). Washington, D.C.: The Mathematical Association of America, Dolciani Mathematical Expositions, 7.

FERRINI-MUNDY, J. y GEUTHER GRAHAM, K. (1991). An Overview of the Calculus Curriculum Reform Effort: Issues for Learning, Teaching and Curriculum Development. The American Mathematical Monthly, 98(7), pp. 627-635.

FERRINI-MUNDY, J. y GAUDARD, M. (1992). Secondary school calculus: preparation or pitfall in the study of college calculus? Journal for Research in Mathematics Education, 23(1), pp. 56-71.

FREUDENTHAL, M. (1973). Mathematics as an educational task. Dordrecht-Holland: D. Reidel Publishing Company.

GONZÁLEZ URBANEJA, P.M. (1992). Las raíces del cálculo infinitesimal en el siglo XVII. Madrid: Alianza Universidad.

GRAS MARTÍ, A., LÓPEZ-GAY, R., MARTÍNEZ TORREGROSA, J. y TORREGROSA, G. (2001). On how to best introduce the concept of differential in physics. First International Girep Seminar Developing Formal Thinking in Physics. University of Udine, Italia. (En prensa).

JHONSON, K. (1995). Harvard Calculus at Oklahoma State University. The American Mathematical Monthly, 102(9), pp. 794-797.

KLEINFELD, M. (1996). Calculus: Reformed or Deformed? The American Mathematical Monthly, 103(3), pp. 230-232.

KLINE, M. (1972). El pensamiento matemático de la antigüedad a nuestros días. Madrid: Alianza Universidad, 1992.

KNISLEY, J. (1997). Calculus: A Modern Perspective. The American Mathematical Monthly, 104(8), pp. 724-727.

LAUGWITZ, D. (1997a). On the Historical Development of Infinitesimal Mathematics. Part I: The algoritmic thinking of Leibniz and Euler. The American Mathematical Monthly, 104(5), pp. 447-455.

LAUGWITZ, D. (1997b). On the Historical Development of Infinitesimal Mathematics. Part II: The conceptual thinking of Cauchy. The American Mathematical Monthly, 104(7), pp. 654-663.

LAUTEN, D., GRAHAM, K. y FERRINI-MUNDY, J. (1994). Student Understanding of Basic Calculus Concepts: Interaction with the Graphics Calculator. Journal of Mathematical Behavior, 13, pp. 225-237.

LAVALY, A. (1990). Do students find physics easier to learn without mathematical problems? Physics Education, 25, pp. 202-204.

LÓPEZ DELOS MOZOS, M.C. (1991). Aproximación didáctica al concepto de derivada. Revista Números, 21, pp. 7-14.

LÓPEZ-GAY, R., MARTÍNEZ TORREGROSA, J. y GRAS MARTI, A. (2001a). What is the meaning and use of this expresion: $d N=\alpha \cdot N \cdot t^{2} \cdot d t$ ? International Conference: Physics Teacher Education Beyond 2000. Selected Contributions. Pintó, R. y Surinach, S. (eds.). París: Elsevier Editions.

LÓPEZ-GAY, R., MARTÍNEZ TORREGROSA, J. y GRAS MARTÍ, A. (2001b). Una propuesta alternativa para mejorar el uso del cálculo diferencial en las clases de física. Diseño experimental y resultados. Enseñanza de las Ciencias, núm. extra. VI Congreso, pp. 335-336.

MARTIN, D. y COLEMAN, J. (1994). Mathematics for mature student access to HE courses in physics: the Coventry perspective. Physics Education, 29, pp. 20-22.

MARTÍNEZTORREGROSA, J., DOMÉNECH, J.L. y VERDÚ, R. (1994). Del derribo de ideas al levantamiento de puentes: la epistemología de la ciencia como criterio organizador 
de la enseñanza en las ciencias física y química. Revista de Enseñanza de la Física, 7(2).

MARTÍNEZ TORREGROSA, J. y LÓPEZ-GAY, R. (1992). La evolución del concepto de diferencial y la comprensión de su significado por profesores y alumnos. International Conference on History of the Physical-Mathematical Sciences and the Teaching of Sciences, pp. 132-136. Madrid: European Physical Society.

MARTÍNEZ TORREGROSA, J. y LÓPEZ-GAY, R. (1993). El uso del concepto de diferencial en la enseñanza de la física. Enseñanza de las Ciencias, número extra. IV Congreso, pp. 259-260.

MARTÍNEZ TORREGROSA, J. y LÓPEZ-GAY, R. (1997a). El uso del cálculo diferencial en la enseñanza de la física y la química en el bachillerato: ¿una ayuda o un obstáculo? Enseñanza de las Ciencias, núm. extra. V Congreso, pp. 397-398.

MARTÍNEZ TORREGROSA, J. y LÓPEZ-GAY, R. (1997 b). La introducción y uso del concepto de diferencial en los textos de física y química en el bachillerato: ¿favorece un aprendizaje con comprensión? Enseñanza de las Ciencias, número extra. V Congreso, pp. 399-400.

MONK, M. (1994). Mathematics in physics education: a case of more haste less speed. Physics Education, 29(4), pp. 209211.

NAGY, P. et al. (1991). High School Calculus: comparing the content of assignments and tests. Journal for Research in Mathematics Education, 22(1), pp. 69-75.

ORTON, A. (1983a). Students' understanding of integration. Educational Studies in Mathematics, 14, pp. 1-18.

ORTON, A. (1983 b). Students' understanding of differentiation. Educational Studies in Mathematics, 14, pp. 235-250.

OSTEBEE, A. y ZORN, P. (1997). Pro Choice. The American Mathematical Monthly, 104 (8), pp. 728-730.
ROSSI, P. (1997). El nacimiento de la ciencia moderna en Europa. Barcelona: Crítica - Grijalbo Mondadori, 1998.

SÁNCHEZ, C. y CONTRERAS, A. (1998). Análisis de manuales a través del tratamiento didáctico dado al concepto límite de una función: una perspectiva desde la noción de obstáculo. Enseñanza de las Ciencias, 16(1), pp. 73-84.

SCHNEIDER, M. (1991). Un obstacle épistémologique soulevé par des «découpages infinis» des surfaces et des solides. Recherches en Didactique des Mathématiques, 11(23), pp. 241-294.

SCHNEIDER, M. (1992). A propos del l'apprentissage du taux de variation instantane. Educational Studies in Mathematics, 23, pp. 317-350.

SWANN, H. (1997). Commentary on Rethinking Rigor in Calculus: The Role of the Mean Value Theorem. The American Mathematical Monthly, 104(3), pp. 241-245.

THOMPSON, P.W. (1994). Images of rate and operational understanding of the Fundamental Theorem of Calculus. Educational Studies in Mathematics, 26, pp. 229-274.

THOMPSON, P.W. y THOMPSON, A. (1994). Talking about rates conceptually. Part I: Teacher's struggle. Journal for Research in Mathematics Education, 25(3), pp. 279-303.

TUCKER, T. (1997). Rethinking Rigor in Calculus: The Role of the Mean Value Theorem. The American Mathematical Monthly, 104(3), pp. 231-240.

TURÉGANO, P. (1998). Del área a la integral. Un estudio en el contexto educativo. Enseñanza de las Ciencias, 16(2), pp. 233-249.

WHITE, P. y MITCHELMORE, M. (1996). Conceptual knowledge in introductory calculus. Journal for Research in Mathematics Education, 27(1), pp. 79-95.

WILLIAMS, S. (1991). Models of limit held by college calculus students. Journal for Research in Mathematics Education, 22(3), pp. 219-236.

[Artículo recibido en enero de 2001 y aceptado en septiembre de 2001.] 